\title{
UN MODELO DE SECADO NATURAL DE EUCALIPTO \\ (Eucalyptus globulus L.)
}

\author{
Rubén A. Ananías (*) \\ Francisco P. Vergara (**)
}

\section{RESUMEN}

Se propone un modelo de secado natural para describir la pérdida de humedad en madera aserrada de eucalipto (Eucalyptus globulus L.) de $25 \mathrm{~mm}$ de espesor.

La madera es expuesta por un año a las condiciones ambientales en un patio de secado natural de una planta industrial de la Octava Región. La pérdida semanal de humedad en la madera es relacionada con las variables climáticas obtenidas de una caseta metereológica especialmente construida.

El modelo predice el tiempo de secado al aire y permite el desarrollo de un calendario tentativo de secado al aire para la zona en estudio.

Palabras claves: Secado natural. Tiempo de secado. Eucalytpus globulus.

\section{ABSTRACT}

An air - drying model is proposed for describing moisture content losses of $25 \mathrm{~mm}$ thick eucalypts (Eucalyptus globulus L.) timber.

The timber is exposed for about one year to the enviroment in an air - drying yard. The weekly rate of timber moisture loss is related with the metereological data measured in a weather station installed in the air - drying yard.

The model predicts air - drying times for wich an air - drying schedule can be developed, for the climatic zone studied.

Keywords: Air - drying time. Eucalyptus globulus.

(") Magister en Tecnologia de la Madera, Profesor Asistente, Departamento Ingenieria en Maderas, Facultad Ingeniería. Universidad del Bio - Bio. Concepción. Chile.

(“) Ingeniero Civil en Industrias Forestales. Asesor Gerencia Producción, Industrias Colcura Ltda. Lota. Chile. 


\section{INTRODUCCION}

La madera aserrada de eucalipto es secada comúnmente mediante un proceso combinado de secado al aire hasta aproximadamente el punto de saturación de la fibra y posteriormente un secado en horno hasta el contenido de humedad final deseado.

La pérdida de humedad durante el secado al aire depende básicamente de las condiciones climáticas, la especie y la escuadría de la madera. Para optimizar esta relación deben tenerse presente los costos asociados a la mantención del inventario y la eventual pérdida en calidad de la madera. Esto significa encontrar el punto de equilibrio entre la disminución del contenido de humedad y el incremento de los costos de secado al aire, lo cual ocurre cuando la pérdida de humedad se reduce fuertemente y presenta un comportamiento irregular.

La determinación en forma regular del contenido de humedad en la madera durante el secado al aire libre se vuelve muchas veces impracticable como consecuencia del grán número de mediciones a ejecutar. Esta necesidad de manejar una gran cantidad de información para conducir en forma adecuada el secado de madera ha llevado a la formulación de diversos modelos matemáticos. En este sentido Dening y Wengert (1982), desarrollan una expresión matemática para estimar el tiempo de secado al aire en función del contenido de humedad inicial y las variables climáticas, demuestran que las precipitaciones y la velocidad del aire tienen un efecto limitado sobre la pérdida de humedad durante el secado de maderas duras con un contenido de humedad inicial bajo el $80 \%$.

El cálculo del tiempo de secado al aire de la madera es influenciado por las características de las variables consideradas, que afectan en cierto grado el rango de validez de las estimaciones. No obstante, se establece generalmente la expresión que se ajusta de la mejor forma al comportamiento de la madera en la situación real. En este trabajo se propone un modelo que predice el tiempo de secado al aire de la madera aserrada de eucalipto radial de $25 \mathrm{~mm}$ de espesor, en la zona de Concepción.

\section{METODOLOGIA}

La madera es obtenida a la salida de la mesa de clasificación del aserradero. Se recogen 24 lingas (paquetes con separadores) de madera que se ubican en el patio de secado en 5 castillos diferentes. Se utilizan 3 rangos de escuadrías de acuerdo con la producción. Los ensayos se inician en verano. 
El comportamiento del contenido de humedad de la madera en secado al aire se mide a través del empleo de muestras testigos de $50 \mathrm{~cm}$ de largo con sus puntas selladas convenientemente. El contenido de humedad inicial de las muestras testigo se determina con probetas extraídas de la misma tabla. Con estos valores y mediante pesadas semanales de las muestras se estima periódicamente la pérdida de humedad.

Una caseta meteorológica construída en el mismo patio de secado al aire, permite obtener las temperturas máxima y mínima y la humedad relativa del ambiente de secado. El registro se efectúa 3 veces al dia de acuerdo a las especificaciones meteorologicas.

Durante un año se estudia la evolución de la pérdida de humedad semanal de la madera sometida al proceso de secado al aire. El resultado de estas observaciones se lleva a una correlación del tipo:

$\mathrm{CH} / \mathrm{t}=\mathrm{f}\left(\mathrm{CHt} ; \mathrm{T}^{\circ} ; \mathrm{H}\right)$

Donde:

$\mathrm{CH} / \mathrm{t}=$ pérdida semanal humedad $(\% /$ semana $)$

$\mathrm{CH} / \mathrm{t}=$ contenido humedad en cualquier instante $\mathrm{t}(\%)$

$\mathrm{T}^{\circ}=$ Temperatura media semanal $\left({ }^{\circ} \mathrm{C}\right)$

$\mathrm{H}=$ Humedad especifica media semanal (\%)

Un análisis de regresión múltiple permite estimar cuál es la relación funcional que predice de mejor forma el comportamiento de la pérdida semanal de humedad en la madera durante el secado al aire libre.

Las predicciones del modelo obtenido permiten la construcción de un calendario de secado y hacer estimaciones del comportamiento de la humedad de la madera en el tiempo. Tales estimaciones presentan limitaciones dadas por la época de inicio, la zona en estudio y el número de observaciones experimentales, puesto que corresponden a un ensayo de secado al aire, entre Febrero y Diciembre de 1988, en cancha de secado de una empresa de la zona de Concepción (Octava Región, Chile).

\section{RESULTADOS Y DISCUSION}

El comportamiento de la pérdida semanal de humedad durante el secado al aire 
de madera aserrada de eucalipto radial de $25 \mathrm{~mm}$ de espesor queda descrito por la relación:

$\mathrm{CH} / \mathrm{t}=-13,0594+0,5256 \mathrm{~T}^{\circ}-0,0426 \mathrm{H}+0,0019 \mathrm{CHt}$

$r^{2}=0,48$.

$\mathrm{CH}, \mathrm{T}^{\circ}, \mathrm{H}$ y CHt definidos anteriormente.

En el Cuadro $N^{\circ} 1$ y Figura $N^{\circ} 1$ se muestra la evolución del secado natural durante un año en base al modelo propuesto. Se presenta la pérdida semanal de humedad para madera aserrada de eucalipto desde un $80 \%$ de humedad inicial. Se observa que las estaciones más frías y húmedas dificultan el secado natural bajo $30 \%$ de humedad y que para alcanzar esta humedad existe una diferencia de 35 dias en favor de las maderas que comienzan a secarse en época de verano.

\section{Cuadro $\mathrm{N}^{\circ} 1$}

\section{CALENDARIO DE SECADO AL AIRE EN LA ZONA DE CONCEPCION PARA MADERA DE EUCALIPTO RADIAL DE 25 MM DE ESPESOR}

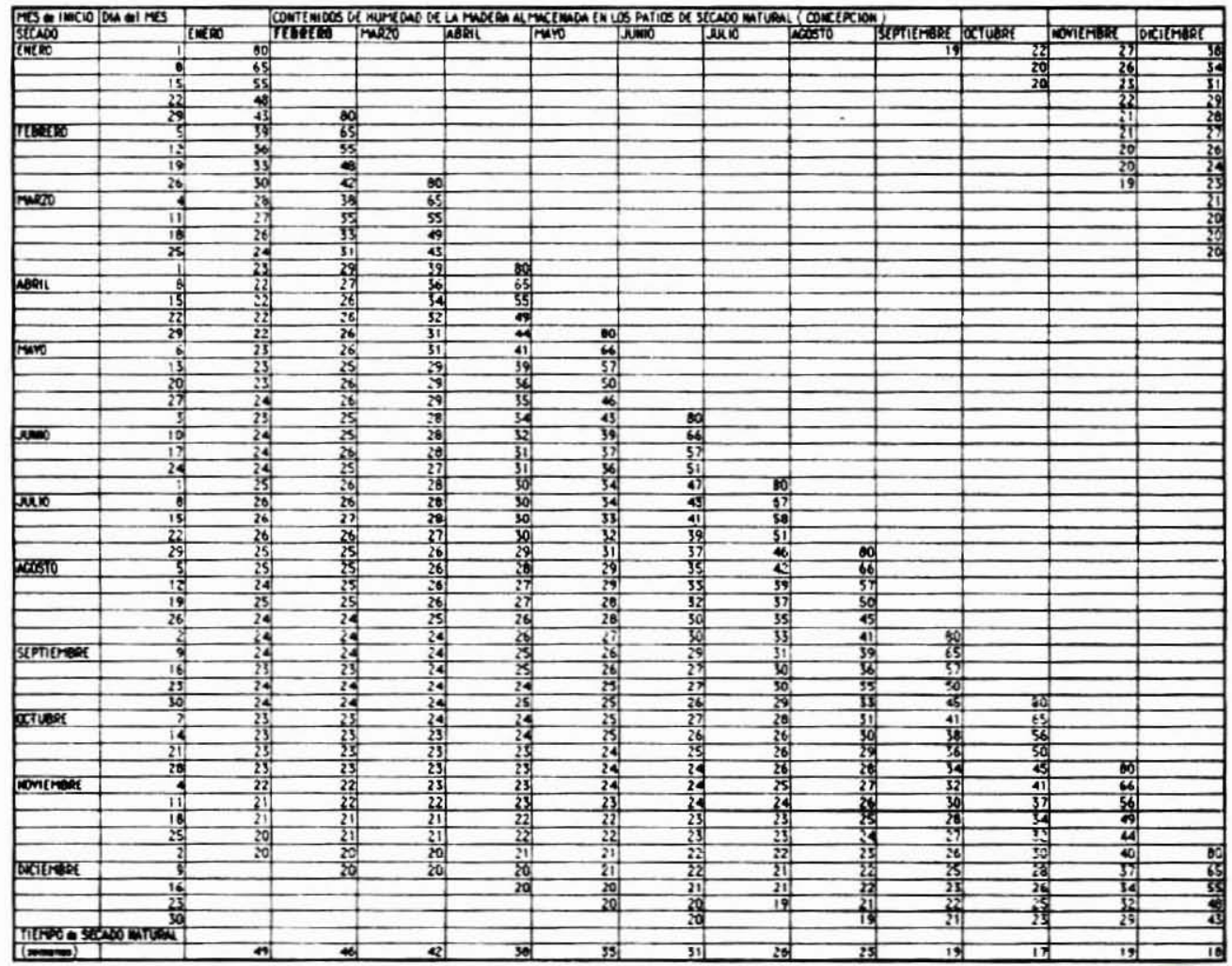




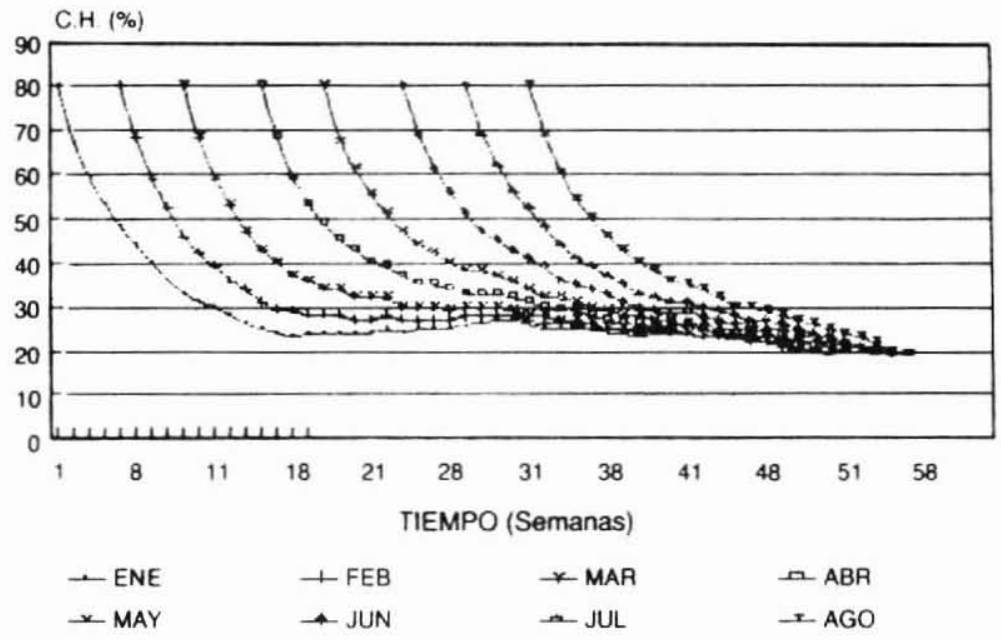

Figura $\mathrm{N}^{\circ} 1$ CURVAS DE SECADO NATURAL ESTIMADAS EN LA ZONA DE CONCEPCION PARA EUCALIPTO RADIAL DE 25 MM DE ESPESOR

En el Cuadro $\mathrm{N}$ '2 se indica la duración del secado al aire del eucalipto en relación a la época de inicio del proceso y al contenido de humedad final deseado. De ahi es posible predecir que existe secado durante toda la época de verano, lo que permite estimar el número de días efectivos de secado para cada mes del año y construir el calendario de secado que se muestra en Cuadro $N^{\circ} 3$.

\section{Cuadro $\mathrm{N}^{\circ} 2$}

TIEMPO ESTIMADO DE SECADO NATURAL EN EUCALIPTO RADIAL DE 25 MM DE ESPESOR

\begin{tabular}{|l|c|c|c|}
\hline Epoca Inicio Secado & \multicolumn{3}{|c|}{ Tiempo (semanas) } \\
\cline { 2 - 4 } & $\mathrm{CH} \mathrm{30 \%}$ & $\mathrm{CH} 25 \%$ & $\mathrm{CH} \mathrm{20 \%}$ \\
\hline Otoño & 14 & 27 & 41 \\
Invierno & 15 & 23 & 31 \\
Primavera & 12 & 15 & 19 \\
Verano & 10 & 13 & 19 \\
\hline
\end{tabular}


El calendario de secado indica 240 días efectivos de secado anual para la especie, el espesor, la localidad y el año de estudio. No obstante, si es conocido el número de dias que requiere para su secado al aire cualquier otra especie o espesor, el calendario de secado puede ser útil, considerando las limitaciones derivadas de las variaciones climáticas y de localización geográfica.

\section{Cuadro $\mathrm{N}^{\circ} 3$}

DIAS EFECTIVOS DE SECADO AL AIRE DURANTE EL AÑO 1988 EN LA ZONA DE CONCEPCION

\begin{tabular}{|l|c|}
\hline Mes & Dias de Secado Mensual \\
\hline Enero & 30 \\
Febrero & 30 \\
Marzo & 25 \\
Abril & 15 \\
Mayo & 10 \\
Junio & 5 \\
Julio & 10 \\
Agosto & 15 \\
Septiembre & 20 \\
Octubre & 25 \\
Noviembre & 25 \\
Diciembre & 30 \\
\hline
\end{tabular}

Por otra parte en la Figura $\mathrm{N}^{\circ} 2$ se observa la curva de secado natural real y estimada, en ambas curvas la pérdida semanal de humedad presenta una tendencia similar. Los datos experimentales para la construcción de la curva de secado real se recogen de ensayos iniciados en época de verano y corresponden a los resultados promedios obtenidos para los 3 rangos de anchos o escuadrías consideradas. De igual forma la curva estimada se construye en base al modelo general propuesto que es independiente del ancho de las piezas, ya que una correlación similar es obtenida para cada ancho de acuerdo con Vergara (1990). El modelo general simplifica en la práctica su operación y aplicaciones. 
C.H. (\%)

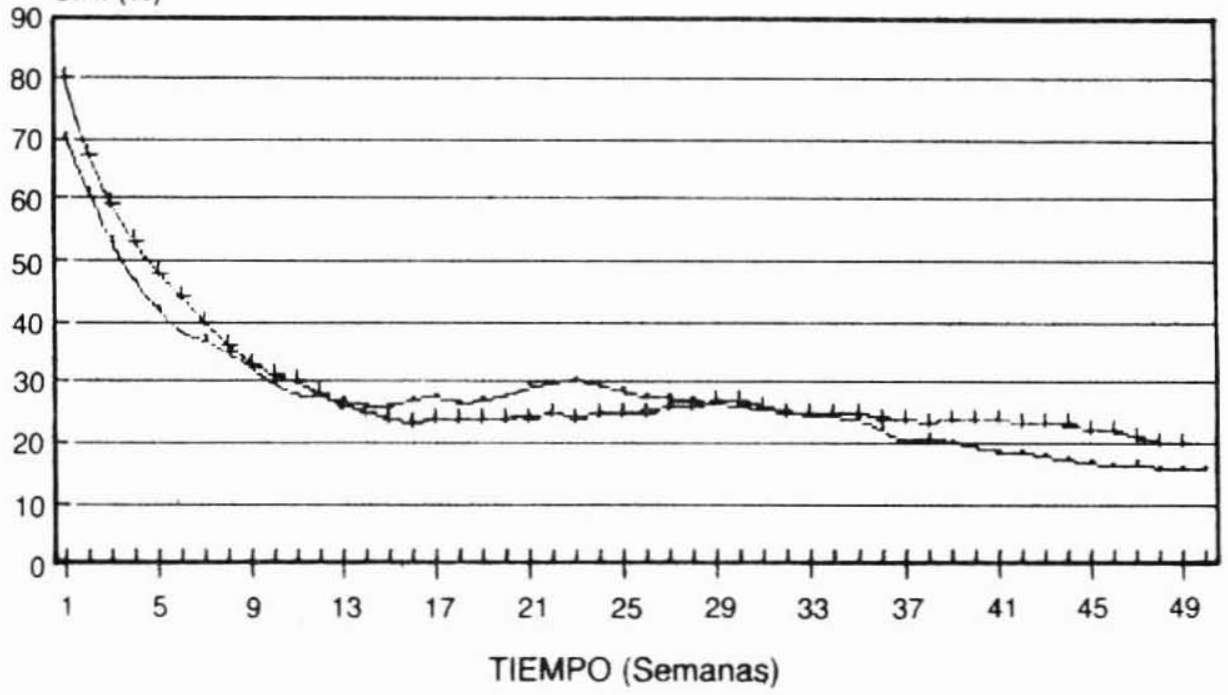

- C.H. Real $\perp$ C.H. Estimado

Figura $N^{\circ} 2$ CURVAS DE SECADO AL AIRE REAL Y ESTIMADA PARA EUCALIPTO RADIAL DE 25 MM DE ESPESOR

\section{CONCLUSIONES}

El modelo de secado al aire propuesto describe el comportamiento de la pérdida semanal de humedad en madera de eucalipto radial de $25 \mathrm{~mm}$ de espesor.

El tiempo de secado estimado para madera de eucalipto radial de $25 \mathrm{~mm}$ de espesor es de 70 días efectivos.

El calendario de secado al aire indica 240 días de secado anual para la especie, según las variables consideradas, esto es espesor, localización geográfica y época de inicio del proceso.

El modelo propuesto es una contribución preliminar para el cálculo del tiempo de secado al aire del eucalipto en cualquier lugar y para cualquier escuadria. 


\section{REFERENCIAS BIBLIOGRAFICAS}

Denig, J.; Wengert, E., 1982. Estimating air-drying moisture content losses for red-oak and yellow - poplar lumber. Forest Prod. J. 32(2):26-31.

Rietz, R., 1972. A calendar for air-drying lumber in the upper midwest. U.S.D.A. Forest Service. Research Note FLP-0224. Madison, Wis.

Vergara, F., 1990. Estudio del secado natural. Determinación de curvas de secado. Modelación del secado natural. Proyecto Titulación, Depto Ingeniería en Maderas, Facultad de Ingeniería, Universidad del Bio-Bio, Concepción, Chile. 